\title{
MEASURING THE CORROSION RATE OF ALUMINUM BY USING ULTRA THIN LAYER ACTIVATION TECHNIQUE
}

\author{
Imam Kambali, Hari Suryanto, Parwanto \\ Cyclotron Division, Center for Development of Radioisotopes and Radiopharmaceuticals \\ National Nuclear Energy Agency, Kawasan Puspiptek Serpong, Tangerang, Indonesia \\ Email: imamkey@batan.go.id; hsuryant@yahoo.com
}

\begin{abstract}
MEASURING THE CORROSION RATE OF ALUMINUM BY USING ULTRA THIN LAYER ACTIVATION TECHNIQUE. Corrosion rate of aluminum ( $99.9 \%$ purity) in $0.001 \mathrm{M}$ hydrochloride acid solution has been measured by using Ultra Thin Layer Activation (UTLA) technique. Iron foil of $25 \mu \mathrm{m}$ thickness was used as the primary target and activated by $10.2 \mathrm{MeV}$ proton beam coming from BATAN's cyclotron with irradiation dose of $0.73 \mu \mathrm{Ah}\left(9.534 \times 10^{15}\right.$ protons $\left./ \mathrm{cm}^{2}\right)$. Radioisotope ${ }^{56} \mathrm{Co}$ formed on the foil would have sufficient kinetic energy to run out of the foil's surface and be implanted on the aluminum used as the secondary target. After 2 days of cooling down, about $0.397 \mathrm{MBq}$ of ${ }^{56} \mathrm{Co}$ stayed on the foil was counted by $\mathrm{NaI}(\mathrm{Tl})$ detector coupled with Multi Channel Analyzer (MCA). It was found that about $9.45 \%$ of the total ${ }^{56} \mathrm{Co}$ formed on the iron foil was successfully implanted into aluminum target. Corrosion test of aluminum was conducted in a 2 inches diameter pipe filled with $0.001 \mathrm{M}$ hydrochloride acid flowing with a rate of 80 liters/minute. The corrosion rate of the aluminum was determined from the change of ${ }^{56} \mathrm{Co}$ activity during the process. The result of experiment indicated that the sample was corroded with a very slow rate of $0.064 \mathrm{~nm} /$ minute and $0.0054 \mathrm{~nm} /$ minute for measuring time of $0-180$ and 180-300 minutes respectively.
\end{abstract}

Keywords : Ultra Thin Layer Activation (UTLA) Technique, corrosion rate, proton beam, cyclotron

\section{INTRODUCTION}

Nowadays various techniques and methods for measuring corrosion, erosion and wear rates of materials and industrial components have been developed. Conventional methods such as gravimetric, thermal microbalance, loss of reflectivity, interference color, metallographic examination, electrical resistance, ultrasonic and other techniques are still applied for special reasons [1]. However, the conventional methods show their weakness in case of low accuracy, precision and sensitivity in performing the result of measurement. Some of the methods need to destruct the material when the measurement is conducted. Therefore, new method based on nuclear technique has been under developed these days. The technique comprises 2 different ways of irradiation process, e.g. Thin Layer Activation (TLA) and Ultra Thin Layer Activation (UTLA). TLA technique employs a beam of charged particles (proton, deuteron, ${ }^{3} \mathrm{He}$ and ${ }^{4} \mathrm{He}$ ) or neutron directly to label a very thin layer (in the order of micrometer) of the material's surface, while UTLA technique labels the material by means of recoil radioisotope implantation (indirect activation). On the other words, there are 2 targets in recoil radioisotope implantation technique; a primary target (to produce a 
certain radioisotope) and a secondary target (material to be analyzed) where recoil radioisotope will be implanted on.

The main advantages of the nuclear (TLA and UTLA) technique are as follows [2]:

1. Non-destructive remote monitoring of surface degradation, including wear, corrosion and erosion.

2. In-situ and on line measurement.

3. Simultaneous measurement of surface degradation of several components in the same machine.

4. High sensitivity in monitoring the slow rate degradation process.

5. No influence on the operating conditions of machine or system.

6. Very low level of radioactivity (about $370 \mathrm{kBq}$ or $10 \mu \mathrm{Ci}$ )

7. Quicker compared to conventional methods.

When a thin target (few micrometers) of elementary composition is bombarded with a beam of charged particles, nuclear reactions generate heavy radioactive atoms acquiring sufficient energy to recoil out of the primary target and be implanted to the material, which is to be a secondary target. This is the basic principle of recoil radioisotope implantation technique. For the purposes of TLA, this method is advantageous when any of the matrixes (trace elements) of the sample cannot be activated properly with the available beam or only an extremely thin active layer is necessary $[2,3,4]$.

Another good point of the UTLA technique is that the depth of penetration (depth profile) of the implanted atoms is very shallow (in the order of nanometer, depends on the implanted atoms and the secondary target) so that a very low rate of corrosion, erosion and wear of material can be detected precisely whenever any other techniques cannot be applied for.

Detroi, F., et al [3] mentioned in their research that ${ }^{56} \mathrm{Co}$ atoms produced from nuclear reaction between a beam of energetic proton and iron foil could be used properly for wear measurement. With the same aims, in 1997, Detroi, F., et al [2] monitored and measured wear of aluminum machine parts and plastic coatings by using recoil implantation method of ${ }^{7} \mathrm{Be}$ radioisotope.

At different place and time, Sauvage, T., et al [5] have also investigated inconel pipes with a very low corrosion rate of $4.5 \mathrm{~nm} /$ month by using recoil radioisotope implantation of ${ }^{56} \mathrm{Co}$ labeled on the sample's surface. The result of the investigation has justified the use of the technique for measuring realtime corrosion rate of materials, which could not be done by using direct activation (TLA) and other techniques.

Based on the references above, it is important to carry out and develop the use of the recoil radioisotope implantation technique for measuring degradation of pure aluminum, which is known to have a very low rate of corrosion. Aluminum ranks second only to iron and steel in the metal 
markets. The rapid growth of aluminum industry is attributed to a unique combination of properties, which makes it one of the most versatile of engineering and construction materials. It is highly corrosion-resistant under a great many service conditions and is non-toxic. The aluminum is often used as coiled tubing and piping installation [1] in which corrosion may occur when chemical solution is flowing in the tube or pipe. In this case, measuring and monitoring corrosion of the aluminum will be an important part of enhancing its reliability.

This experiment aims mainly to measure the corrosion rate of aluminum so that its life assessment can be determined precisely in order to avoid unwanted accident during the process. In this experiment, ${ }^{56} \mathrm{Co}$ would be employed as the implanted atom (dopant atom) and its depth profile would be used as calibration curve for measuring corrosion rate of aluminum sample in a solution of $0.001 \mathrm{M}$ hydrochloric acid flowing with a rate of 80 liters/minute. Meanwhile the required proton beam was extracted from the fixed-energy proton beam produced by BATAN's cyclotron. In order to decrease the proton's energy from $26.5 \mathrm{MeV}$ to $10.2 \mathrm{MeV}$, an aluminum foil of $1.477 \mathrm{~mm}$ thickness was set as degrader.

\section{THEORETICAL REVIEW}

When a high energy proton (more than $5 \mathrm{MeV}$ ) interacts with iron or aluminum atoms, nuclear reaction will take place as follows $[2,10]$ :

$$
\begin{array}{ll}
{ }^{56} \mathrm{Fe}+{ }^{1} \mathrm{p} \longrightarrow & \left(\mathrm{T}_{1 / 2}=78.8 \text { days }\right) \\
{ }^{27} \mathrm{Al}+{ }^{16} \mathrm{Co}+{ }^{1} \mathrm{n} & \left(\mathrm{T}_{1 / 2}=4.16 \text { seconds }\right)
\end{array}
$$

The main characteristic of material activation is the thick target yield $(Y)$, which is determined as the radionuclide activity $(A)$ per irradiation unit of the beam current $(I)$ at the end of irradiation [2]:

$$
Y=\frac{A}{I} \frac{\lambda}{1-e^{-\lambda t}}
$$

where $t$ is irradiation time and $\lambda$ is decay constant.

The thick target yield always depends on the energy of the incident ion or particle, which penetrates through a certain range after losing its whole energy. The range of the ion $(R)$, with energy $E$, in $x \mu \mathrm{m}$ thickness target is simply defined as $[6,7,8]$ :

$$
R=\int_{0}^{R} d x=\frac{1}{N} \int_{0}^{E_{0}} \frac{d E}{S_{n}(E)+S_{e}(E)}
$$


In this expression, $N$ is density of target, $S_{n}$ is nuclear loss and $S_{e}$ is electronic loss.

When an energetic ion interacts with a thin target, recoil atom or ion will be produced and may run out of the target's surface with kinetic energy $T$. The kinematical relationships of the interaction can be expressed as follows [6]:

$$
T=\frac{2 E}{\left(1+\frac{M_{2}}{M_{1}}\right)}\left[\frac{M_{2}}{M_{1}}+\operatorname{Sin}^{2} \theta-\operatorname{Cos} \theta \sqrt{\left(\frac{M_{2}}{M_{1}}\right)^{2}-\operatorname{Sin}^{2} \theta}\right]
$$

where $M_{1}$ is mass number of incident ion, $M_{2}$ is mass number of recoil atom and $\theta$ is the scattering angle of ion. Equation (2) and (3) can be calculated quickly by using SRIM-96 software [9].

A very important characteristic of a label is the depth distribution of radionuclide. It represents the calibration curve used for conversion the activity change into the depth of the layer removed. Experimental calibration curve is more precise. It can be obtained either by the stack of foil technique or by abrasion of the standard. The calibration curve is generally described by polynomial of power $m[2]$ :

$$
y=\sum_{k=0}^{m} a_{k} x^{k}
$$

where $x$ represents the depth of penetration of the recoil radionuclide, $y$ is relative activity of the radionuclide, while $k, a$, and $m$ are constants.

Abrasion of standard is good way of calibration when an ultra thin radioactive layer is formed. Eventually, the degradation process caused by corrosion, erosion and wear can be determined by measuring the remaining activity of the implanted radioisotope in the target after exposing the sample in a corrosive environment for a period of time.

\section{EXPERIMENTAL}

\section{Sample Preparation}

Iron foil (99.5\% purity) of $25 \mu \mathrm{m}$ thickness and $2 \mathrm{~cm}$ diameter was prepared for primary target, while a $1.5 \mathrm{~cm}$ diameter aluminum $(99.9 \%$ purity) of $3 \mathrm{~mm}$ thickness was used for secondary target. In order to clean and move the impurity atoms away from the surface of each sample, alcohol was used as cleaner solution. 


\section{Irradiation and Implantation Process}

Figure 1 illustrates the experimental set-up for UTLA Technique. A $10.2 \mathrm{MeV}$ proton beam of $0.73 \mu \mathrm{Ah}$ dose $\left(9.534 \times 10^{15}\right.$ protons $\left./ \mathrm{cm}^{2}\right)$ was produced by BATAN's cyclotron. In order to decrease the proton's energy from initial value of $26.5 \mathrm{MeV}$ to $10.2 \mathrm{MeV}$, an aluminum foil of $1.477 \mathrm{~mm}$ thickness was set as degrader. Aluminum collimator of $1.5 \mathrm{~cm}$ diameter was also set to focus the proton beam. The extracted beam was bombarded to the prepared-iron foil of $1.5 \mathrm{~cm}$ diameter until radioactive layer of ${ }^{56} \mathrm{Co}$ was formed on the most part of the foil. The radioactive atoms of ${ }^{56} \mathrm{Co}$ would have sufficient kinetic energy to run out of the iron's surface and be implanted on the aluminum target. A distance of $1.8 \mathrm{~cm}$ long separated the iron foil and aluminum. Since the implantation process was conducted in non-vacuum chamber, aluminum window was placed at the end of the beam line to separate the vacuum and non-vacuum chamber. The integrated current of ${ }^{56} \mathrm{Co}$ beam was measured by using a simplified Faraday cup. Radioactivity of ${ }^{56} \mathrm{Co}$ on each target was counted by using $\mathrm{NaI}(\mathrm{Tl})$ detector coupled with MCA (Multi Channel Analyzer) made by AMPTEK-USA after 2 days of cooling down. Meanwhile the stopping power and range of $10.2 \mathrm{MeV}$ proton beam and ${ }^{56} \mathrm{Co}$ in the air and aluminum were simulated independently by SRIM-96 software for preliminary calculation.

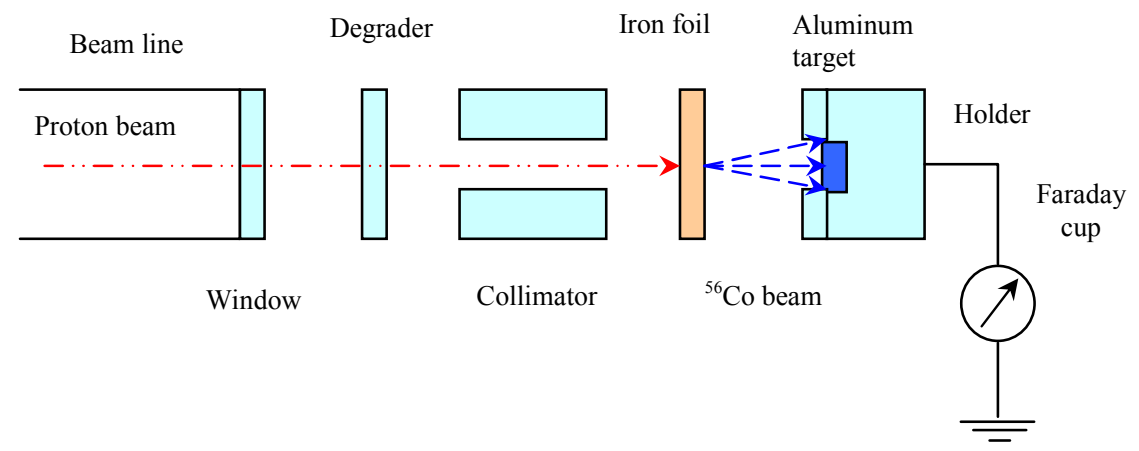

Figure 1. Experimental set-up for UTLA technique

\section{Determination of Calibration Curve}

Experimental calibration curve (depth profile) of the implanted ${ }^{56} \mathrm{Co}$ in aluminum was done by abrasion of the standard sample using abrasive paper of 1000 mesh. The relative radioactivity of ${ }^{56} \mathrm{Co}$ in a certain depth of aluminum was measured by $\mathrm{NaI}(\mathrm{Tl})$ detector, while the thickness of the abraded surface was converted from the weight measurement. By using a simple equation stated the relation between the specific mass $(\rho)$ and the area 
of the specimen $\left(\mathrm{L}_{\mathrm{A}}\right)$, its thickness $(\mathrm{t})$ could be calculated theoretically as indicated in equation below:

$$
t=\frac{m}{\rho \cdot L_{A}}
$$

where $m$ is the mass of the abraded sample.

SATORIUS GMBH analytical balance of type 2434 was used for the weight measurement. The analytical balance could be used to weigh a sample which has a weight in the order of microgram. Eventually, the relation between relative activity and depth profile of the implanted ${ }^{56} \mathrm{Co}$ in aluminum was plotted by Microsoft Excel program to determine the calibration curve.

\section{Corrosion Test and Measurement}

In order to test the corrosion rate of aluminum, the specified sample of $99.9 \%$ purity was mounted on the coupon holder so that its surface was at the same line as the inner surface of the piping installation. The sample was then exposed in a 2 inches diameter pipe filled with $0.001 \mathrm{M}$ hydrochloride acid solution flowing with a rate of 80 liters/minute. In this corrosive environment, aluminum would be corroded in a very slow rate. The corrosion rate was determined from the change of ${ }^{56} \mathrm{Co}$ activity in every 60 minutes during the process. This could be done by the help of calibration curve. The measurement consumed totally 330 minutes.

\section{RESULT AND DISCUSSION}

Irradiation of $25 \mu \mathrm{m}$ iron foil by $10.2 \mathrm{MeV}$ proton beam may cause the most part of the foil to become radioactive. Radioisotope ${ }^{56} \mathrm{Co}$ is produced in this nuclear reaction since only $5 \mathrm{MeV}$ proton's energy is required to activate the iron atoms [2]. This means the rest energy of $5.2 \mathrm{MeV}$ would be the kinetic energy of ${ }^{56} \mathrm{Co}$ atom, as it left the foil. Radioisotope ${ }^{56} \mathrm{Co}$ decays with a relatively short half-life of 78.8 days and emits 4 different gamma ray energies as shown in Table 1.

Table 1. Gamma energies emitted from ${ }^{56} \mathrm{Co}$ atom [2]

\begin{tabular}{|c|c|}
\hline Gamma energy (keV) & Relative probability \\
\hline 847 & 1,0 \\
\hline 1038 & 0,14 \\
\hline 1238 & 0,68 \\
\hline 1272 & 0,16 \\
\hline
\end{tabular}


Since the most intensive gamma rays are at the energy of $847 \mathrm{keV}$ and $1238 \mathrm{keV}$, the gamma spectroscopy system used in this experiment could measure only 2 peaks of the energies, while the two other peaks could not be detected properly due to their lower probability. As shown in Figure 1, there is also a gamma ray of $511 \mathrm{keV}$. It may be the annihilation peak of gamma ray energy of higher than $1.02 \mathrm{MeV}$, where pair production interaction can take place [7]. However, for the purpose of corrosion, erosion and wear rate measurement the energy of $847 \mathrm{keV}$ would be the best choice because of its perfect decay fraction.

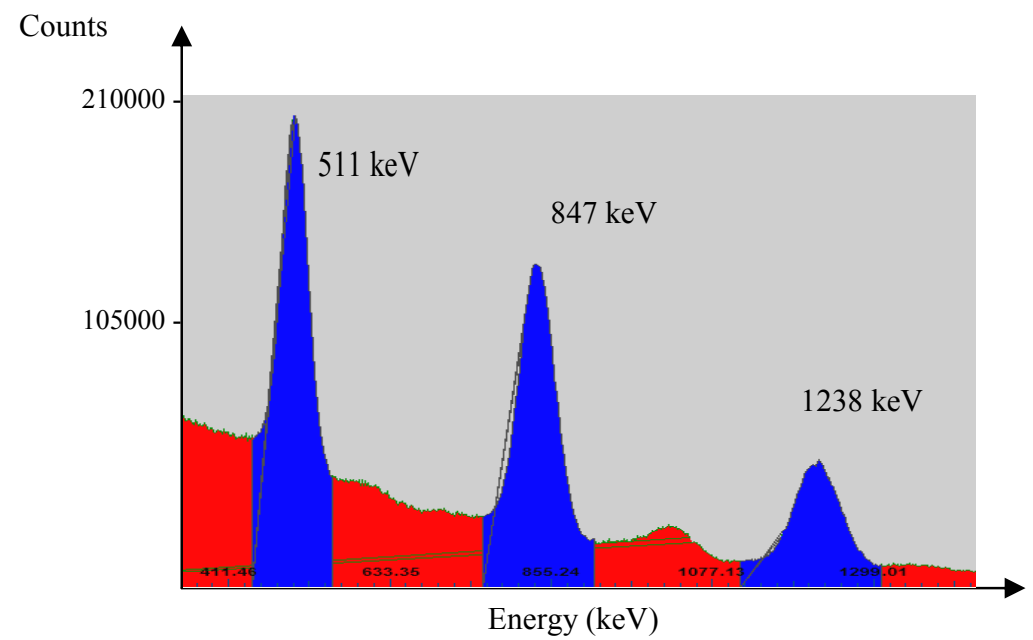

Figure 1. Measured energy spectra for ${ }^{56} \mathrm{Co}$ in iron foil as primary target.

Following the activation, an activity of $0.397 \mathrm{MBq}$ of ${ }^{56} \mathrm{Co}$ atoms was measured on the iron foil after 2 days of cooling time. In this case, it gives a dose rate of only $0.116 \mu \mathrm{Sv} /$ hour from the distance of $1 \mathrm{~m}$, which is relatively safe for the measuring personnel. For practical purposes, this dose should be related to common situation. For instance, a chest x-ray gives a total dose of $20 \mu \mathrm{Sv}$ [10]. Clearly, the personnel would need to spend 172.4 hours at $1 \mathrm{~m}$ distance to receive the same dose as a chest $\mathrm{x}$-ray. This means there was no credible risk of exposure to staff working in the vicinity.

By using a simulation of SRIM-96 software, a total kinetic energy of about $5.2 \mathrm{MeV}$ owned by ${ }^{56} \mathrm{Co}$ is used for leaving the foil. Tracking on $1.8 \mathrm{~cm}$ long of the air might cause ${ }^{56} \mathrm{Co}$ lose its most energy, so that only a small fraction of $150 \mathrm{keV}$ would be the rest energy as it passed through the aluminum. It would consequently penetrate only a very shallow depth of the aluminum's surface. This ultra thin radioactive layer is a great benefit as a label since the detector can detect a very small activity change caused by abrasion of the layer. The total activity of ${ }^{56} \mathrm{Co}$ counted in the $\mathrm{Al}$ target was $0.02 \mathrm{MBq}$. 
Implanted ${ }^{56} \mathrm{Co}$ atoms were measured by gamma spectroscopy system in order to assure that the recoil implantation was occurred. Figure 2 shows the measured energy spectra of implanted ${ }^{56} \mathrm{Co}$. Nearly $9.45 \%$ of the total ${ }^{56} \mathrm{Co}$ formed on the iron foil was successfully implanted into aluminum target. Other spectra excluding ${ }^{56} \mathrm{Co}$ spectra were also measured. It was identified as ${ }^{27} \mathrm{Si}$, which was created from the interaction between proton beam and the aluminum target. This interaction may occur since there is a possibility of proton beam leakage. On the other word, a certain fraction of the proton beam may penetrate through the iron foil without any significant interaction and eventually reach the aluminum to create radioisotope ${ }^{27} \mathrm{Si}$. However this very short-lived radionuclide (4.16 seconds half life) [11] did not contribute significantly to the counting process because its activity would had rapidly closed to zero when the corrosion measurement was carried out.

In order to make correction of the activity caused by ${ }^{27} \mathrm{Al}(\mathrm{p}, \mathrm{n})^{27} \mathrm{Si}$ reaction, a pure aluminum was irradiated independently without recoil implantation of ${ }^{56} \mathrm{Co}$ into $\mathrm{Al}$ target. In this case, after 2 days of cooling down, the activity of ${ }^{27} \mathrm{Si}$ would be zero and its spectra would not appear on the display of MCA.

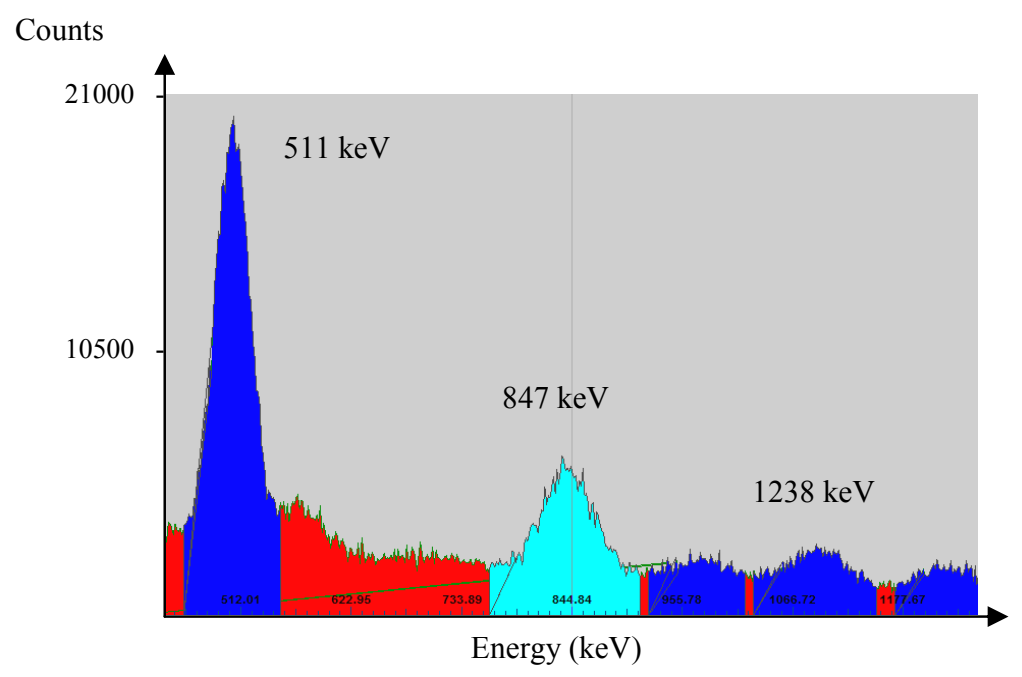

Figure 2. Measured energy spectra for ${ }^{56} \mathrm{Co}$ implanted in aluminum as secondary target.

Experimental calibration curve, which represents the depth distribution or depth profile of the actual recoil ${ }^{56} \mathrm{Co}$ implanted in aluminum, was conducted by a very simple and precise way of abrasion the standard sample, which has exactly the same chemical composition with the studied sample. Abrasive paper of 1000 mesh was used for the abrasion process. Every abraded layer was weighed by analytical balance and then converted to surface loss of the removed layer. If the relation between relative activity of 
the implanted ${ }^{56} \mathrm{Co}$ and its depth of penetration is plotted from the data of experiment, the calibration curve will be acquired. Figure 3 performs the calibration curve acquired in this experiment. It is described by the polynomial of power 4 , where $y=3 \times 10^{-8} x^{4}-9 \times 10^{-6} x^{3}+0.0009 x^{2}-0.0455 x+$ 0.9384. This equation is plotted with $\mathrm{R}^{2}=0.9931$, which is understood to have a high confidential level.

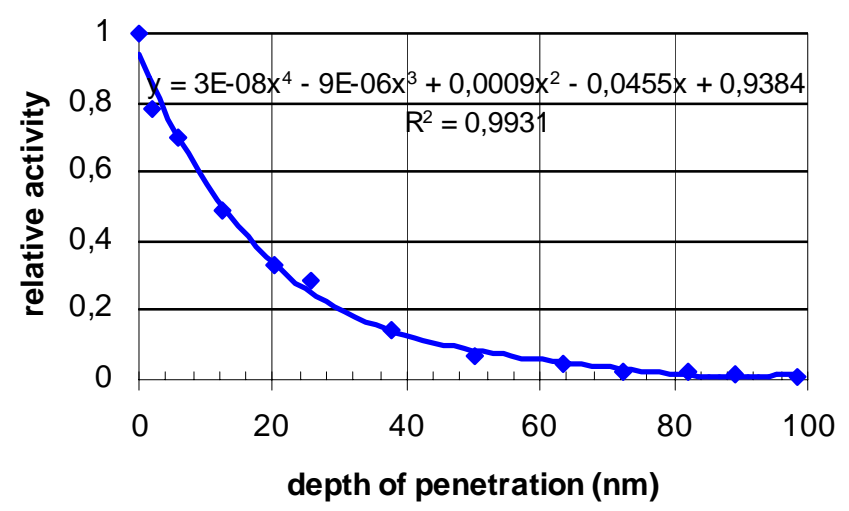

Figure 3. Depth profile (calibration curve) of ${ }^{56} \mathrm{Co}$ implanted in aluminum $(99.9 \%)$.

It is clear from Figure 3 above that the maximum range of the implanted ${ }^{56} \mathrm{Co}$ into aluminum is in an order of nanometer, which is less than $100 \mathrm{~nm}$. This extremely shallow range indicates that a very small change of the radionuclide activity due to the abrasion process will be detected precisely by gamma spectroscopy system. The calibration curve was then employed to help measuring the corrosion rate of aluminum sample by assuming that the corrosion process was followed by erosion process (removal of the activated layer). On the other word, this calibration curve is applicable as long as there is abrasion or erosion of the aluminum's surface.

After exposing in $0.001 \mathrm{M}$ hydrochloride acid solution flowing with a rate of 80 liters/minute, the data of the experiment was plotted to determine the corrosion rate of the sample. The corrosion kinetic of the aluminum is shown in Figure 4, which indicates a very slow rate of corrosion. The trendline of the corrosion kinetic is $y=-9 \times 10^{-5} x^{2}+0.0452 x+0.6218$ with a regression coefficient of $\mathrm{R}^{2}=0.9168$. 


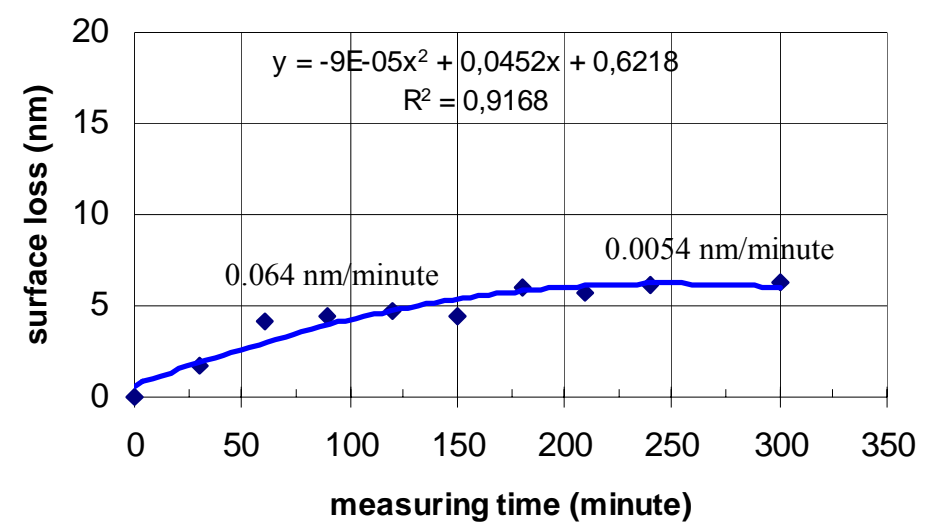

Figure 4. Corrosion of aluminum in $0.001 \mathrm{M}$ hydrochloride acid solution and with a flow rate of 80 liters/minute.

At the beginning of the corrosion process, the sample was corroded with a rate of $0.064 \mathrm{~nm} /$ minute. It occurred for measuring time of $0-180$ minutes. Meanwhile, for measuring time of 180-300 minutes the rate was extremely slow with a nominal value of $0.0054 \mathrm{~nm} /$ minute. As shown in Figure 4, up to 180 minutes of exposing time, the rate increases rapidly, which signs that the formation of protective oxide layer remains slow. However, after 180 minutes exposure, its rate become low and the corrosion curve tends to flat. This phenomenon indicates that a protective oxide layer was already formed on the aluminum's surface so that it could prevent further corrosion. As predicted before, the corrosion was generally followed by erosion of the activated layer. In this case, the calibration curve made from the experimental data is applicable for this measurement.

If the corrosion rate of the sample remains $0.0054 \mathrm{~nm} /$ minute for measuring time of longer than 330 minutes, its life assessment can be determined from this experiment. Since the thickness of the aluminum sample was $3 \mathrm{~mm}$, the sample would resist for a relatively long time of less than 560.000 minutes (1.08 years).

It has been proven experimentally that the recoil radioisotope implantation technique is a proper nuclear method in measuring corrosion rate of aluminum and other materials, where the rate is extremely slow (in nanometer scale) and any other techniques cannot be applied for. This measurement does not waste times to get sufficient data for the analysis. For the next experiment, it is important to use some lighter and heavier radioactive atoms like ${ }^{7} \mathrm{Be},{ }^{22} \mathrm{Na},{ }^{48} \mathrm{~V},{ }^{51} \mathrm{Cr},{ }^{56} \mathrm{Ni}$ and ${ }^{65} \mathrm{Zn}$ as dopant atoms. This experiment will justify the most proper dopant atoms used as a radioactive label. In addition, the experiment on several different targets 
(non metals, plastics and ceramics) with various parameters of implantation will be useful for achieving the result of further experiments perfectly.

\section{CONCLUSION}

1. In $0.001 \mathrm{M}$ hydrochloride acid solution of 80 liters/minute flow rate, aluminum is corroded with a very low rate of $0.064 \mathrm{~nm} /$ minute and $0.0054 \mathrm{~nm} /$ minute for measuring time of $0-180$ and 180-300 minutes respectively.

2. If the corrosion rate of the sample remains $0.0054 \mathrm{~nm} /$ minute for measuring time of longer than 330 minutes, the sample will resist for a relatively long time of less than 560.000 minutes (1.08 years).

\section{ACKNOWLEDGEMENT}

We greatly thank to Mr. Subardi, Mr. Parwanto and Mr. Edi Slamet for their assistances in operating the cyclotron, installing the targets and expanding the mechanical components of recoil radioactive implantation technique.

\section{REFERENCES}

1. FONTANA, M.G., "Corrosion Engineering", $3^{\text {rd }}$ edition, McGraw Hill Book Company, Singapore (1987).

2. ANONYMOUS, "The Thin Layer Activation Method and Its Applications in Industry", IAEA-TECDOC-924, Vienna, Austria (1997).

3. DITRÓI, F., TARKÁNYI, F., MAHUNKA, I., Nuclear Implantation of Radioactive Atoms by Using Secondary Target on Cyclotron Beams for Thin Layer Activation, Institute of Nuclear Research, Debrecen, Hungary (1997).

4. DITRÓI, F., MAHUNKA, I., Thin Layer Activation with recoil implantation, Institute of Nuclear Research, Debrecen, Hungary (1997).

5. SAUVAGE, T., VINCENT, L., BLONDIAUX, G., Thin Layer Activation (TLA) and Ultra Thin Layer Activation (UTLA): 2 Complementary Techniques For Wear and Corrosion Studies in Various Fields, CNRS CERI, France.

6. MILOSEVSKY, G.V., "Monte Carlo Numerical Simulation of Ion Implantation Into Solid Targets", Luikov Heat and Mass Transfer, Institute of the National Academy of Sciences of Belarus, The Radiative Gas Dynamics Laboratory, (2000). 
7. KNOLL, G.F., "Radiation Detection and Measurement", second edition, John Willey and Sons, Singapore (1989).

8. ANONYMOUS, Ion Implantation, dunham.ee.washington.edu/ ee539/ notes/ chapter8.pdf (1999).

9. ZIEGLER, J.F., The Stopping and Range of Ions in Matter, WwW.srim.org.

10. YANG, F., HAMILTON, J.H., "Modern Atomic and Nuclear Physics", The McGraw-Hill Companies, Inc., Singapore (1996).

11. WALlACE, G., TLA in a Coal-Fired Power Station, National Isotope centre, Institute of Geological and Nuclear Sciences Ltd, New Zealand (2002). 